\section{ORIGINAL RESEARCH}

\author{
E. Nyberg \\ G.S. Sandhu \\ J. Jesberger \\ K.A. Blackham \\ D.P. Hsu \\ M.A. Griswold \\ J.L. Sunshine
}

\title{
Comparison of Brain MR Images at 1.5T Using BLADE and Rectilinear Techniques for Patients Who Move during Data Acquisition
}

\begin{abstract}
BACKGROUND AND PURPOSE: MR imaging of moving patients can be challenging and motion correction techniques have been proposed though some have associated new artifacts. The objective of this study was to semiquantitatively compare brain MR images of moving patients obtained at $1.5 \mathrm{~T}$ by using partially radial and rectilinear acquisition techniques.
\end{abstract}

\begin{abstract}
MATERIALS AND METHODS: FLAIR, T2-, T1-, and contrast-enhanced T1-weighted image sets of 25 patients (14-94 years) obtained by using BLADE (like PROPELLER, a partially radial acquisition) and rectilinear techniques in the same imaging session were compared by 2 neuroradiologists in terms of extent of the motion artifact, image quality, and lesion visibility. ICC between opinions of the evaluators was calculated.
\end{abstract}

\begin{abstract}
RESULTS: Of the total of 70 image sets, the motion artifact was small in the partially radial images in 43 and in the rectilinear images in 13, and the opinions of the evaluators were discordant in the remaining 14 sets $(I C C=0.63, P<.05)$. The quality of partially radial images was higher for 36 sets versus 9 rectilinear sets, with disagreement between the 2 evaluators in the remaining 25 (ICC $=0.15$, $P<$.05). Pathologic lesions were better characterized on 37 sets of partially radial images versus 13 sets of rectilinear images, and opinions of the evaluators differed in 20 sets (ICC $=0.90, P<.05$ ). The neuroradiologists deemed 4 sets of rectilinear images nondiagnostic compared with only 1 set of radial images.
\end{abstract}

CONCLUSIONS: The data demonstrate that our application of BLADE sequences reduces the extent of motion artifacts in brain images of moving patients, improving image quality and lesion characterization.

ABBREVIATIONS: $\mathrm{CE}=$ contrast-enhanced (or postcontrast); ICC $=$ intraclass correlation coefficient; PROPELLER = periodically rotated overlapping parallel lines with enhanced reconstruction

$\mathbf{M}$ $\mathrm{R}$ imaging of moving patients is a challenging task because motion artifacts in MR images degrade their quality and clinical utility. ${ }^{1}$ A number of motion correction techniques have been proposed to overcome this challenge. ${ }^{2-5}$ One such technique is PROPELLER — and, similarly, BLADE - in which MR signal intensity is collected by using a multishot TSE sequence. Multiple echo trains of this sequence are used to sample the $k$-space points in the form of partially overlapping strips called blades. These blades are arranged in a radial fashion, with each blade running through the center of the $k$-space with a width proportional to the echo-train length. ${ }^{6-8}$ The number of the blades and echo-train length are user specified. The location of each blade is rotated around the center of the $k$-space, with subsequent repetition times. Each overlapping blade thus samples the center of the $k$-space, resulting in redundant data, and this redundancy is used to compare data strips for any inconsistencies that occurred within the overlapping region of the $k$-space as a result of in-plane rotation and translational head motion. These phase inconsistencies

Received February 28, 2011; accepted after revision May 9.

From the Department of Radiology (E.N., G.S.S., K.A.B., M.A.G., J.L.S., D.P.H.), University Hospitals, Case Center for Imaging Research and Departments of Physics and Biomedical Engineering (M.A.G.), Case Western Reserve University, Cleveland, Ohio.

Research sponsored by Siemens Medical Solutions and Case Center for Imaging Research. Please address correspondence to Jeffrey L. Sunshine MD, PhD, Department of Radiology, Bolwell B 123, 11100 Euclid Ave, Cleveland, OH 44106; e-mail: Jeffrey. Sunshine@uhhospitals.org

http://dx.doi.org/10.3174/ajnr.A2737 are then corrected before reconstruction of the final image. ${ }^{8}$ Additionally, this partially radial acquisition technique is relatively insensitive to susceptibility- and eddy-currentrelated ${ }^{9-11}$ pulsation and Gibb artifacts. ${ }^{12}$ However, such techniques can lengthen the data acquisition time and introduce a type of wrap, or radial artifact, into the MR images, particularly those obtained in coronal or sagittal orientations, which has been described in the literature. ${ }^{12}$ The influence of these artifacts on the clinical utility of images obtained with these techniques has yet to be investigated.

Clinically, BLADE/PROPELLER-type techniques may be used to obtain brain MR images of various contrasts in moving and nonmoving patients, and previous investigators have shown that the quality of images generated with these techniques from cooperative patients is comparable to those obtained by using the rectilinear technique. ${ }^{12}$ Limited data available in the literature also suggest improved image quality from this technique in pediatric patients ${ }^{7,13,14}$ and with diffusion imaging on moving adult patients. ${ }^{6}$ We semiquantitatively assessed the impact of such partially radial acquisition imaging on image quality in moving patients, who were imaged for a variety of indications at our tertiary care center, by using multiple pulse sequences. We compared the images obtained with this technique with the images of corresponding pulse sequences obtained by using conventional rectilinear techniques. We hypothesized that BlADE/PROPELLER techniques would produce fewer artifacts, improve overall image quality, and support characterization of pathologic brain le- 
Table 1: The imaging sequence, sequence parameters, and total scan time for acquisition of T2WI, FLAIR, T1WI, and CE-T1WI images by partially radial and rectilinear techniques

\begin{tabular}{|c|c|c|}
\hline Image Type & $\begin{array}{l}\text { BLADE (Imaging sequence/TR/TE/ } \alpha / \mathrm{TI} / \mathrm{FOV} / \text { section } \\
\text { thickness/base resolution/blade coverage/motion } \\
\text { correction/turbo factor/echo-train length per section/ } \\
\text { averages/concatenation/imaging time) }\end{array}$ & $\begin{array}{c}\text { Rectilinear (Imaging sequence/TR/TE/ } \alpha / \mathrm{TI} / \mathrm{FOV} / \\
\text { section thickness/base resolution/ } \\
\text { averages/concatenation/imaging time) }\end{array}$ \\
\hline $\mathrm{T} 2 \mathrm{WI}$ & $\begin{array}{l}\text { TSE/4000 ms/107 ms/150\%/-/230 } \times 230 \mathrm{~mm} / 5 \mathrm{~mm} / 256 \times \\
\text { 256/91.7\%/on/35/11/1/2/1 minute } 38 \text { seconds }\end{array}$ & $\begin{array}{l}\text { TSE/4320 ms/88 ms/150\% } /-/ 173 \times 230 \mathrm{~mm} / 5 \mathrm{~mm} / 192 \\
\times 256 / 1 / 1 / 1 \text { minute } 13 \text { seconds }\end{array}$ \\
\hline FLAIR & $\begin{array}{l}\text { FLAIR/9000 ms/107 ms/150\%/150 ms/230 × } 230 \mathrm{~mm} / 5 \mathrm{~mm} / \\
256 \times 256 / 91.7 \% / \mathrm{on} / 35 / 11 / 1 / 2 / 3 \text { minutes } 38 \text { seconds }\end{array}$ & $\begin{array}{l}\text { FLAIR/4320 ms/110 ms/150\%/2500 ms/173 × } 230 \mathrm{~mm} / \\
5 \mathrm{~mm} / 192 \times 256 / 1 / 1 / 1 \text { minute } 21 \text { seconds }\end{array}$ \\
\hline Axial T1WI & $\begin{array}{l}\text { FLAIR/2500 ms/59 ms/150\%/860 ms/230 × } 230 \mathrm{~mm} / 5 \mathrm{~mm} / \\
256 \times 256 / 95.5 \% / \mathrm{off} / 19 / 21 / 1 / 3 / 2 \text { minutes } 47 \text { seconds }\end{array}$ & $\begin{array}{l}\text { Spin-echo/740 ms/17 ms/90\%/-/173 × } 230 \mathrm{~mm} / 5 \mathrm{~mm} / \\
192 \times 256 / 2 / 1 / 4 \text { minutes } 8 \text { seconds }\end{array}$ \\
\hline Coronal T1WI & $\begin{array}{l}\text { FLAIR/2500 ms/59 ms/150\%/860 ms/230 × } 230 \mathrm{~mm} / 5 \mathrm{~mm} / \\
256 \times 256 / 95.5 \% / \mathrm{off} / 19 / 21 / 1 / 5 / 4 \text { minutes } 37 \text { seconds }\end{array}$ & $\begin{array}{l}\text { Spin-echo/740 ms/17 ms/90\%/-/201 × } 230 \mathrm{~mm} / 5 \mathrm{~mm} / \\
224 \times 256 / 1 / 1 / 1 \text { minute } 23 \text { seconds }\end{array}$ \\
\hline Sagittal T1WI & $\begin{array}{l}\text { FLAIR/2500 ms/59 ms/150\%/860 ms/230 × } 230 \mathrm{~mm} / 5 \mathrm{~mm} / \\
256 \times 256 / 95.5 \% / \mathrm{off} / 19 / 21 / 1 / 3 / 2 \text { minutes } 52 \text { seconds }\end{array}$ & $\begin{array}{l}\text { Spin-echo/525 ms/17 ms/90\%/-/230 × } 230 \mathrm{~mm} / 5 \mathrm{~mm} / \\
192 \times 256 / 1 / 1 / 1 \text { minute } 52 \text { seconds }\end{array}$ \\
\hline
\end{tabular}

sions in patients with motion degradation, as apparent to acquiring technologists on routine images.

\section{Materials and Methods}

This study is Health Insurance Portability and Accountability Actcompliant and was approved by our Institutional Review Board. The study population consisted of those 25 patients (mean age 60.2 years, range 14-94 years; male/female, 11/14), prospectively collected over 5 consecutive weeks, whose head moved during brain MR data acquisition. MR technologists were instructed to repeat each rectilinear sequence by using the radial BLADE technique (Siemens Medical Solutions, Erlangen, Germany) at their discretion when sufficient motion artifact was noticed during acquisition that, in their opinion, produced readily apparent image degradation. If motion began part way through the MR examination, BLADE was run only after nontrivial motion was detected; no data were reused for multiple comparisons. The imaging protocol of individual patients depended upon their clinical indication and consisted of a varying combination of axial T2WI TSE, T2WI FLAIR, and multiplanar T1WI and CE-T1WI MR images. MR images of these patients were obtained by using both rectilinear and partially radial techniques in the same setting by using similar imaging protocols. Rectilinear pulse sequences were obtained according to the imaging routine at our institution, and these were followed by acquisition by using BLADE for that particular sequence type. This yielded a total of 70 sets of images, 1 set obtained by using the rectilinear technique and the complementary set obtained by using a partially radial technique.

\section{Image Acquisition}

The examinations were performed on 1 of the $1.5 \mathrm{~T}$ scanners (Magnetom Avanto or Espree; Siemens Medical Solutions, Erlangen, Germany) available at our institution and the data were acquired by using a 12-channel head coil (Siemens Medical Solutions). Imaging parameters used to acquire images of each contrast with rectilinear and BLADE techniques, and total imaging times of those sequences, are shown in Table 1, with oversampling by a factor of 2 used during partially radial type acquisitions. A dose of $0.1 \mathrm{mMol} / \mathrm{Kg}$ of Gadoversetamide (Optimark; Mallinckrodt, St. Louis, Missouri) was intravenously administered to obtain contrast-enhanced brain images at our institution.

\section{Image Analysis}

All image annotations were removed to anonymize the images and remove acquisition parameters. Two board-certified neuroradiolo- gists (termed "evaluators" henceforth) separately analyzed the images on off-line workstations. Before image analysis, the evaluators were trained by conducting a practice session on 5 sets of images not part of the study population, and grading standards were agreed upon. The images were evaluated for the presence and degree of motion and radial artifacts, overall image quality, and lesion characterization. First, the sets of images from each sequence and technique were randomized and evaluated individually. Each set was evaluated in terms of the severity of motion artifact, presence of a radial artifact, and visibility of any pathologic lesion. Each set was assigned a motion artifact score by using the following scale: 0 , no visible motion artifact; 1 , mild motion artifact with mild degradation of the image quality; 2 , visible motion artifact with moderate degradation of the image quality; 3 , visible motion artifact with severe degradation of the image quality; 4 , visible motion artifact which renders the image of no diagnostic value. Evaluators then identified any radial artifact in the images and determined whether it compromised the diagnostic yield. Finally, each evaluator identified any pathologic lesion in the image and determined whether the lesion was adequately characterized.

Next, each set of images was paired with its complementary sequence and evaluated with each acquisition technique side-by-side. For this pair-wise comparison, 2 images from the same position of each set were displayed side-by-side in a manner that supported paired scrolling through the image sets. There was random selection of the left-right position of rectilinear or partially radial images between comparisons. Each evaluator then determined which image set a) contained greater motion artifact, b) was of better quality, and c) provided more useful information for characterization of the lesion.

\section{Data Analysis}

The average value of the motion artifact scores assigned by the 2 evaluators was determined for every stack of images. The mean value and standard deviation of the average scores of the stacks of each contrast type, and for all the stacks, was separately calculated for BLADE and rectilinear techniques. The significance of the difference between motion artifact rating scores for the 2 techniques was determined by using a fixed-factors ANOVA test. Effect sizes were Cohen partial eta squared $\left(\eta_{\mathrm{p}}{ }^{2}\right)$ and the effect sizes of $0.21-0.50,0.51-0.80$, and $\geq 0.81$ were considered as a small, moderate, and large effect, respectively.

The images were also analyzed for any detected artifacts that appeared from use of these partially radial-type trajectories, given the radial component in their design. Total numbers of image stacks with any radial artifact, with radial artifact that compromised diagnostic 
Table 2: The mean value and standard deviation of the motion artifact scores for each sequence and overall rating scores from the images obtained using rectilinear and partially radial acquisition techniques

\begin{tabular}{llr}
\hline & \multicolumn{2}{c}{ Motion Score } \\
Image & \multicolumn{2}{c}{ Mean Value \pm Standard Deviation } \\
\cline { 2 - 3 } Type & Rectilinear & \multicolumn{1}{c}{ BLADE } \\
\hline FLAIR & $1.21 \pm 0.63$ & $0.82 \pm 0.87$ \\
T2WI & $1.18 \pm 0.86$ & $0.425 \pm 0.65$ \\
T1WI & $1.83 \pm 1.21$ & $1.57 \pm 1.18$ \\
CE-T1WI & $0.91 \pm 0.64$ & $0.97 \pm 0.62$ \\
Overall & $1.26 \pm 0.89$ & $0.90 \pm 0.92$
\end{tabular}

Motion artifact scoring scale: 0 indicates no visible motion artifact; 1 , visible motion artifact with mild degradation of the image quality; 2 , visible motion artifact with moderate degradation of the image quality; 3 , visible motion artifact with severe degradation of the image quality; 4, visible motion artifact which renders the image of no diagnostic value.

yield, and sequences in which there was a discrepancy between readers for these characteristics were analyzed separately per sequence type and section orientation. Significance of the differences between 2 techniques for radial artifacts was investigated by using an ANOVA test. The relationship between the presence of radial artifact and the image contrast type, as well as the imaging plane for T1WI and CET1WI BLADE image sets, was also determined by using an ANOVA test. The degree of interaction between the reconstruction technique and the sequence for the rating scores was investigated in a post hoc analysis.

The number of FLAIR, T2WI, T1WI, and CE-T1WI image stacks for which opinions of the 2 evaluators matched for detection of a pathologic lesion was separately determined for each type of acquisition. The total number of sets in which 1 technique was preferred over the other, or in which opinions of the evaluators were discrepant, was separately determined for the extent of motion artifact, image quality, and lesion characterization. The corresponding values for T2WI FLAIR, T2WI TSE, T1WI, and CE-T1WI sets were also determined. For all the studied parameters, the degree of agreement between the evaluators was determined by calculating the ICC.

\section{Results}

Seventy sets of images were acquired, including 19 FLAIR, 20 T2WI, 15 T1WI, and 16 CE-T1WI sequences. Of the 31 combined T1WI and CE-T1WI sets, 10 were in axial and 21 were in nonaxial orientation.

There was more motion artifact with the rectilinear technique (higher mean value of scores for motion in the unpaired analyses) for FLAIR, T2WI, and T1WI images. Only for CET1WI images was there a slightly higher mean score for motion with the partially radial technique (Table 2); however, this difference was not significant. The overall motion artifact scores were significantly higher for the rectilinear compared with the partially radial acquisition technique $\left(\eta_{\mathrm{p}}{ }^{2}=0.040\right.$, $P=.021$ ). There was good agreement between the evaluators regarding motion artifact ratings (ICC $0.817, P<.05$ ). Eleven sets of images were scored as having severe motion artifact with both rectilinear and radial acquisition. However, with such motion in these 11,4 sets were further determined to be nondiagnostic with rectilinear acquisition, whereas, despite such motion, only 1 partially radial acquisition set was scored as nondiagnostic.

In review of the rectilinear images, no radial artifact was observed in 67 of the 70 sets. For the remaining 3 stacks, the 2 evaluators had different opinions; however, in none was the diagnostic yield compromised. In contrast, out of 70 partially radial acquisition image sets, radial artifact was observed in 32, not observed in 22, and there was disagreement between the evaluators for the remaining 16 sets of images. Evaluators agreed that the diagnostic yield remained unaffected for 59 sets but that these artifacts resulted in compromise of the diagnostic yield in 9 sets, and 1 evaluator felt yield was compromised in 2 additional sets (moderate interobserver agreement, ICC $=0.67, P<.05$ ). All 11 of these sets were nonaxial T1WI sequences. The incidence of radial artifact was significantly higher for partially radial acquisition than for the rectilinear technique $\left(\eta_{\mathrm{p}}{ }^{2}=0.602, P<.0001\right)$, for all T1WI compared with FLAIR and T2WI sequences $\left(\eta_{\mathrm{p}}{ }^{2}=0.308, P<.0001\right)$, and for nonaxial in contrast to axial T1WI sequences $\left(\eta_{\mathrm{p}}{ }^{2}=0.281\right.$, $P<.0001)$. Radial artifact was found to compromise the diagnostic yield exclusively within the nonaxial images $\left(\eta_{\mathrm{p}}{ }^{2}=\right.$ $0.175, P<.0001$ ) (Table 3 ).

The 2 evaluators were in agreement regarding the presence of a pathologic lesion on 61 partially radial and 63 rectilinear image sets, with disagreement for the remaining 9 and 7 image sets, respectively. The agreement between the evaluators for the presence of a pathologic lesion was moderate (ICC $=0.63$, $P<.05)$. The level of agreement was lower for T1WI and CE-T1WI images for both the techniques.

In the analyses with side-by-side direct comparison between images from the different acquisition techniques and the same sequence contrast, the evaluators identified less motion artifact in the partially radial technique for 43 sets and in the rectilinear technique for 13 sets, with evaluator discrepancy for the remaining 14 sets (agreement level moderate; ICC $=0.63, P<.05)$. The number of patients with greater motion artifact in the partially radial acquisition images was higher for T1WI and lower for FLAIR, T2WI, and CE-T1WI sets (Fig 1A). The evaluators reported better image quality with partially radial acquisition for 36 sets, with rectilinear acquisition for 9 sets, and there was evaluator disagreement for the remaining 25 sets (agreement level low; ICC $=0.15, P<$ $.05)$. Pathologic lesions were better characterized with the partially radial acquisition in 37 sequences, compared with only 13 rectilinear sequences, with evaluator discrepancy in the remaining 20 sets (agreement level good; ICC $=0.90, P<.05$ ). There was a trend toward a higher number of sets with relatively better image quality as well as lesion characterization with the partially radial acquisition for all 4 pulse sequences (Fig $1 B$ and $C$ ).

\section{Discussion}

Our results support our hypothesis, as use of BLADE acquisition techniques did reduce motion artifact and improve image quality in a diverse patient population with overt motion on routine images. These data show that benefits of the BLADE/ PROPELLER-type technique, when applied to multiple pulse sequence types and compared with typical rectilinear acquisitions, can salvage clinical value from scans that may otherwise be rendered nondiagnostic. Similarly, lesion characterization trends were better with partially radial acquisition for all sequence types, including CE-T1WI, despite the presence of more radial artifact (Fig 2). Taken in total, these data demonstrate improved quality and clinical utility of the images when 
Table 3: Total number of axial and non-axial T1WI and CE-T1WI with radial artifact for rectilinear and BLADE techniques and the number of images in which radial artifact compromised the diagnostic yield

\begin{tabular}{|c|c|c|c|c|c|c|c|c|}
\hline \multirow{2}{*}{\multicolumn{2}{|c|}{$\begin{array}{l}\text { Gridding and } \\
\text { Section Orientation }\end{array}$}} & \multirow{3}{*}{$\begin{array}{c}\text { Total } \\
10\end{array}$} & \multicolumn{3}{|c|}{ Radial Artifact } & \multicolumn{3}{|c|}{ Diagnostic Yield } \\
\hline & & & \multirow{2}{*}{$\frac{\text { Absent }}{9}$} & \multirow{2}{*}{$\begin{array}{c}\text { Raters Disagreed } \\
1\end{array}$} & \multirow{2}{*}{$\frac{\text { Present }}{0}$} & \multirow{2}{*}{$\begin{array}{c}\text { Not Compromised } \\
10\end{array}$} & \multirow{2}{*}{$\begin{array}{c}\text { Raters Disagreed } \\
0\end{array}$} & \multirow{2}{*}{$\frac{\text { Compromised }}{0}$} \\
\hline Rectilinear & Axial & & & & & & & \\
\hline & Nonaxial & 21 & 20 & 1 & 0 & 21 & 0 & 0 \\
\hline & Total & 31 & 29 & 2 & 0 & 31 & 0 & 0 \\
\hline \multirow[t]{3}{*}{ BLADE } & Axial & 10 & 1 & 4 & 5 & 10 & 0 & 0 \\
\hline & Nonaxial & 21 & 0 & 1 & 20 & 10 & 2 & 9 \\
\hline & Total & 31 & 1 & 5 & 25 & 20 & 2 & 9 \\
\hline
\end{tabular}
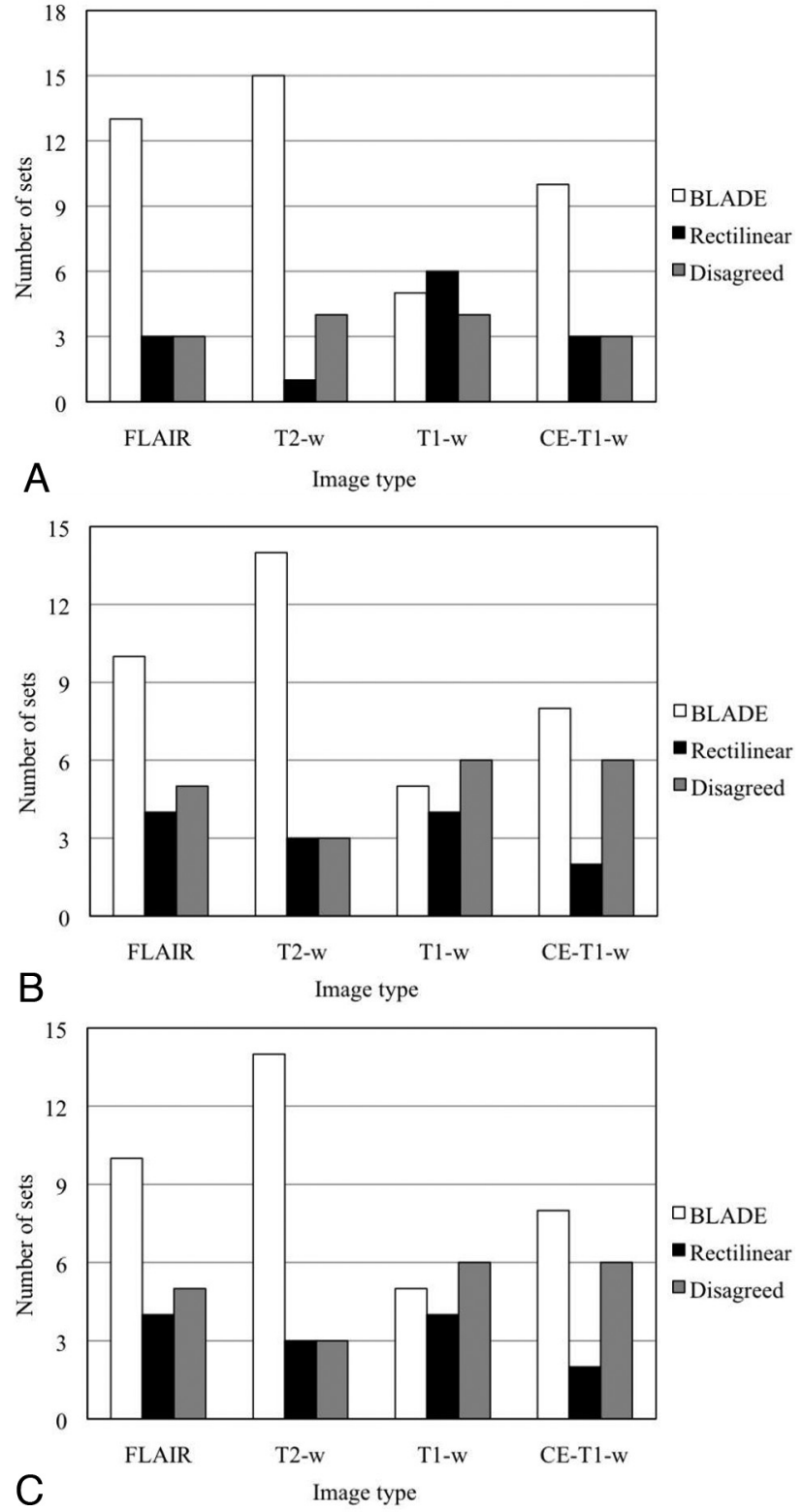

Fig 1. Number of image sets in which the evaluators preferred 1 kind of image over the other or disagreed in terms of $(A)$ less motion artifact, $(B)$ better lesion characterization, and (C) better overall image quality.

this BLADE acquisition technique is applied to patients whose motion is sufficient to otherwise clearly degrade image quality from routine rectilinear sequences.

Improvements were strongest with T2WI and FLAIR images and weaker for T1WI (Figs 3 and 4) even though use of the BLADE/PROPELLER-type technique introduces some radial artifacts on T1WI, primarily in the nonaxial planes. Radial artifact was observed primarily in T1WI and CE-T1WI and became sufficient to compromise the diagnostic utility of the images obtained only in the coronal and sagittal orientations. Our analyses showed that both T1 weighting and nonaxial section orientation independently contributed to radial artifacts. Post hoc analysis, in light of this result, showed a statistically significant interaction specific to nonaxial T1WI $(P<.00001)$. Reviewer scoring shows slight nonsignificant increase in motion on contrast-enhanced images compared with images from the rectilinear technique. Though of uncertain etiology, this may represent a greater conspicuity and altered appearance of pulsation artifacts from enhanced blood in vessels on images from BLADE/PROPELLER-type acquisition and reconstruction.

Our results in a typically encountered adult population with difficulty holding still are concordant with those of previous investigations in cooperative or pediatric groups in showing that the use of BLADE/PROPELLER can produce some artifact but could reduce motion artifact. The results of 1 study evaluating axial T2WI FLAIR BLADE images of cooperative patients obtained at $3 \mathrm{~T}$ demonstrated that radial $k$-space data improved image quality when compared with rectilinear sampling. ${ }^{12}$ In that study, the rectilinear technique was preferred over BLADE for sagittal T1WI due to wrapping of neck and body structures located outside the field of view. However, in those cases where a ghosting or motion artifact was observed in T1WI, the partially radial acquisition technique was preferred. ${ }^{12}$ When compared with the rectilinear technique, BLADE improved lesion visibility and reduced motion artifacts in T2WI FLAIR images of children with neurofibromatosis type $1 .^{14} \mathrm{~T} 2 \mathrm{WI}$ of children obtained by the PROPELLER technique, by using a fast spin-echo sequence, have proved to be of better quality and have lower motion artifacts when compared with their rectilinear counterparts. ${ }^{13}$ Benefits of BLADE/PROPELLER techniques may come with the cost of increased data acquisition time to obtain the required overlapping strips. Our total imaging time was longer for BLADE/ PROPELLER for all sequences except axial T1WI, likely due to a lower number of acquisitions employed. A number of strategies, such as a decrease in the number of blades, ${ }^{10}$ parallel imaging, ${ }^{15,16}$ novel field-of-view strategies, ${ }^{17-19}$ and Turboprop, ${ }^{20}$ are now being explored to reduce the imaging time.

Our study was limited by the variations in imaging parameters for T1WI and T2WI for BLADE and rectilinear acquisitions. However, these were selected as the best standard sequences used in our clinical setting. We also did not compare the 2 techniques in terms of other artifact types, such as pul- 

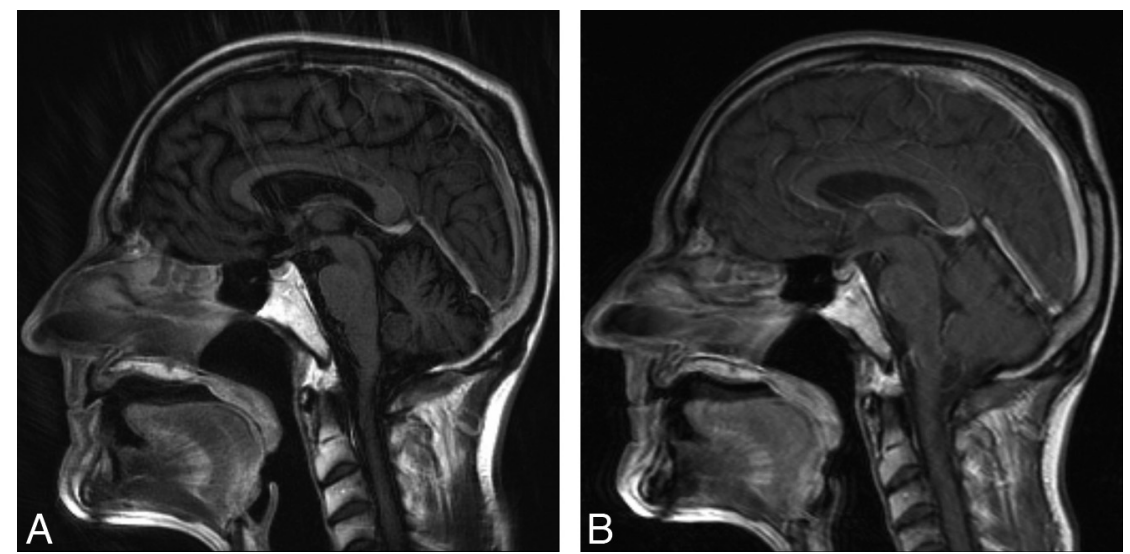

Fig 2. Sagittal CE-T1WI images of a patient obtained by using $(A)$ BLADE and $(B)$ rectilinear technique. Despite the presence of extensive radial artifact in the partially radial acquisition image, gray-white differentiation and correction of motion artifact remain superior.
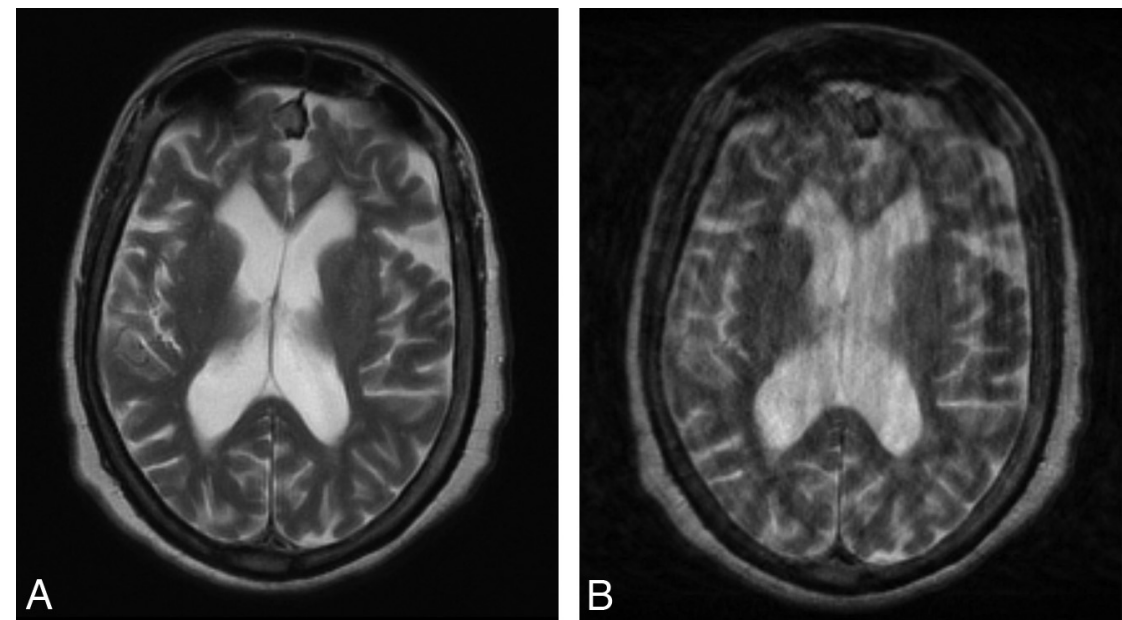

Fig 3. Axial T2WI images of a patient obtained by using $(A)$ BLADE and $(B)$ rectilinear techniques. The partially radial acquisition image is free of motion artifact, whereas extensive motion artifact degrades the rectilinear image.
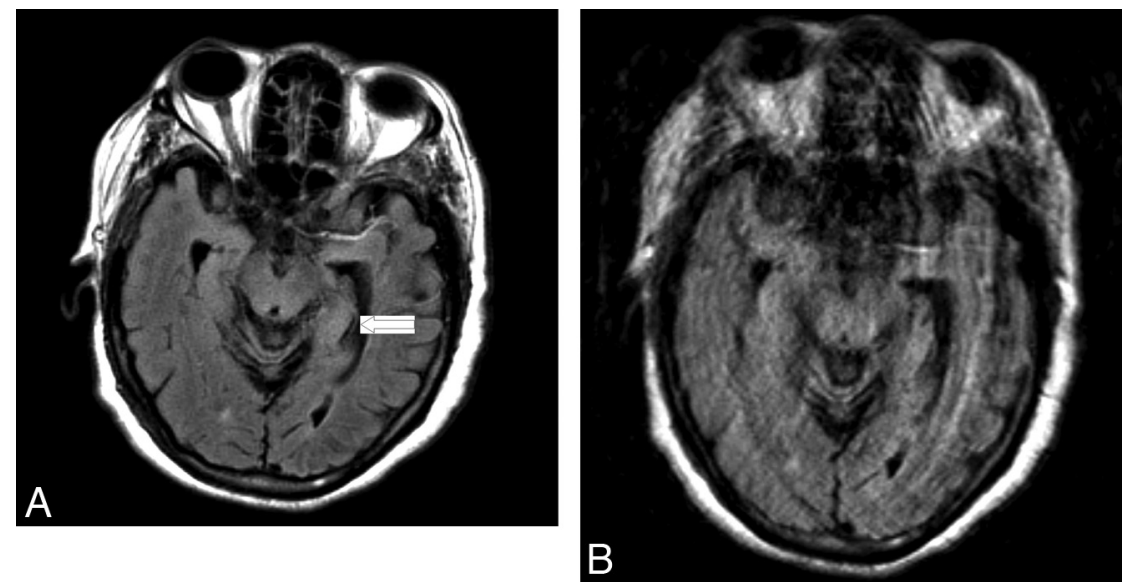

Fig 4. Axial FLAIR images of patient who presented with an acute left middle cerebral artery distribution stroke obtained by using $(A)$ BLADE and $(B)$ rectilinear techniques. The partially radial acquisition image is free of motion artifact, whereas extensive motion artifact degrades quality of the rectilinear image. The small, subtle ischemic lesion was also better characterized from the partially radial acquisition image (arrow).

sation, field inhomogeneity, and eddy-current-related artifacts, though previous reports suggest that BLADE/PROPELLER acquisition is relatively insensitive to these artifacts. ${ }^{9-12}$ An additional prospective study could be performed to further verify these trends.

\section{Conclusions}

Our application of BLADE in moving patients reduced the degree of motion artifacts and improved image quality and lesion characterization, particularly for T2WI and FLAIR images. Use of these motion-correction sequences salvaged diag- 
nostic yield more often than typical rectilinear techniques. The use of a partially radial technique did introduce some radial artifacts in T1WI and CE-T1WI, though the diagnostic yield was preserved in the large, most especially in the axial, plane.

Disclosures: Mark A. Griswold—Research Support (including provision of equipment or materials): Siemens, Details: I am a PI on a sponsored research grant from Siemens. Jeffrey Sunshine-Research Support (including provision of equipment or materials): Siemens, Details: Research grant for MR development that includes some salary support, software, and MR hardware.

\section{References}

1. Murphy KJ, Brunberg JA. Adult claustrophobia, anxiety and sedation in MRI. Magn Reson Imaging 1997;15:51-54

2. Glover GH, Pauly JM. Projection reconstruction techniques for reduction of motion effects in MRI. Magn Reson Med 1992;28:275-89

3. Ehman RL, Felmlee JP. Adaptive technique for high-definition MR imaging of moving structures. Radiology 1989;173:255-63

4. Sachs TS, Meyer $\mathrm{CH}$, Irarrazabal $\mathrm{P}$, et al. The diminishing variance algorithm for real-time reduction of motion artifacts in MRI. Magn Reson Med 1995;34:412-22

5. Qin L, van Gelderen P, Derbyshire JA, et al. Prospective head-movement correction for high-resolution MRI using an in-bore optical tracking system. Magn Reson Med 2009;62:924-34

6. Forbes KP, Pipe JG, Karis JP, et al. Improved image quality and detection of acute cerebral infarction with PROPELLER diffusion-weighted MR imaging. Radiology 2002;225:551-55

7. Forbes KP, Pipe JG, Karis JP, et al. Brain imaging in the unsedated pediatric patient: comparison of periodically rotated overlapping parallel lines with enhanced reconstruction and single-shot fast spin-echo sequences. AJNR Am J Neuroradiol 2003;24:794-98
8. Pipe JG. Motion correction with PROPELLER MRI: application to head motion and free-breathing cardiac imaging. Magn Reson Med 1999;42:963-69

9. Mahmoud OM, Tominaga A, Amatya VJ, et al. Role of PROPELLER diffusion weighted imaging and apparent diffusion coefficient in the diagnosis of sellar and parasellar lesions. Eur J Radiol 2010;79:420-7

10. Arfanakis K, Tamhane AA, Pipe JG, et al. k-space undersampling in PROPELLER imaging. Magn Reson Med 2005;53:675-83

11. Pipe JG, Farthing VG, Forbes KP. Multishot diffusion-weighted FSE using PROPELLER MRI. Magn Reson Med 2002;47:42-52

12. Wintersperger BJ, Runge VM, Biswas J, et al. Brain magnetic resonance imaging at 3 Tesla using BLADE compared with standard rectilinear data sampling. Invest Radiol 2006;41:586-92

13. Vertinsky AT, Rubesova E, Krasnokutsky MV, et al. Performance of PROPELLER relative to standard FSE T2-weighted imaging in pediatric brain MRI. Pediatr Radiol 2009;39:1038-47

14. von Kalle T, Blank B, Fabig-Moritz C, et al. Reduced artefacts and improved assessment of hyperintense brain lesions with BLADE MR imaging in patients with neurofibromatosis type 1. Pediatr Radiol 2009

15. Skare S, Newbould RD, Nordell A, et al. An auto-calibrated, angularly continuous, two-dimensional GRAPPA kernel for propeller trajectories. Magn Reson Med 2008;60:1457-65

16. Chuang TC, Huang TY, Lin FH, et al. PROPELLER-EPI with parallel imaging using a circularly symmetric phased-array RF coil at $3.0 \mathrm{~T}$ : application to high-resolution diffusion tensor imaging. Magn Reson Med 2006;56:1352-58

17. Larson PE, Lustig MS, Nishimura DG. Anisotropic field-of-view shapes for improved PROPELLER imaging. Magn Reson Imaging 2009;27:470-79

18. Devaraj A, Pipe JG. Elliptical field-of-view PROPELLER imaging. Magn Reson Med 2009;62:808-14

19. Deng J, Larson AC. Multishot targeted PROPELLER magnetic resonance imaging: description of the technique and initial applications. Invest Radiol 2009;44:454-62

20. Pipe JG, Zwart N. Turboprop: improved PROPELLER imaging. Magn Reson Med 2006;55:380-85 